\title{
Prevalence of Latent Tuberculosis Infection among Medical Students in South Korea
}

\author{
Da Ho Jung*, Kyung-Wook Jo, M.D.*, Tae Sun Shim, M.D.
}

Division of Pulmonary and Critical Care Medicine, Asan Medical Center, University of Ulsan College of Medicine, Seoul, Korea

Background: We investigated the prevalence of latent tuberculosis infection (LTBI) among medical students in South Korea.

Methods: Students from one medical school, who were in second- or third-year classes before clerkship course, were enrolled for three consecutive years in the study. A standard questionnaire was given to each participant, and tuberculin skin test (TST), QuantiFERON-TB GOLD In-Tube (QFT-GIT) assay, and chest radiography were performed.

Results: A total of 153 participants were enrolled in the study. The mean age of the subjects was $21.9 \pm 0.9$ years, 105 (68.6\%) were male, and 132 (86.3\%) had been vaccinated with Bacille Calmette-Guérin (BCG). Four students $(2.6 \%)$ had a history of contact with tuberculosis (TB) patients during medical practice. No abnormal chest radiograph findings were found for any of the subjects. Of the 153 subjects, 23 (15.0\%) tested positive for the TST, and $8(5.2 \%)$ tested positive for the QFT-GIT. The agreement between the two tests was determined to be 0.34 using kappa coefficients. Of the four students who had a history of contact with TB patients, only one subject tested positive for both tests, and the other three students tested negative for both tests.

Conclusion: A low prevalence of LTBI was found among medical students before clerkship course in South Korea.

Key Words: Latent Tuberculosis; Tuberculin Test; Interferon-gamma Release Tests, Schools, Medical; Students

\section{Introduction}

Tuberculosis (TB) still remains a serious health threat in South Korea, an intermediated TB-burden country. In 2011, 39,557 cases (80.7 cases per 100,000 populations) were officially reported by the Korean Tuberculosis Surveillance system ${ }^{1}$, which is the highest incidence among Organization for Economic Cooperation and Development (OECD) countries.

Address for correspondence: Tae Sun Shim, M.D.

Division of Pulmonary and Critical Care Medicine, Asan Medical Center, University of Ulsan College of Medicine, 388-1, Pungnap-dong, Songpa-gu, Seoul 138-736, Korea Phone: 82-2-3010-3892, Fax: 82-2-3010-6968

E-mail: shimts@amc.seoul.kr

*Da Ho Jung and Kyung-Wook Jo contributed equally to this work.

Received: Aug. 17, 2012

Revised: Sep. 8, 2012

Accepted: Sep. 27, 2012

(a) It is identical to the Creative Commons Attribution Non-Commercial License (http://creativecommons. org/licenses/by-nc/3.0/).
In order to reduce TB burden in South Korea, various measures such as private-public-mix cooperation for TB care and control have been introduced recently ${ }^{2}$. One of the cornerstones of such measures involves targeted screening of subjects who are at high risk of developing TB, followed by treatment of the latent TB infection (LTBI). In addition to the tuberculin skin test (TST), which has been used for more than 100 years for the diagnosis of LTBI, whole blood interferon-gamma releasing assays (IGRAs) based on the Mycobacterium tuberculosis-specific antigens have been introduced recently to diagnose the $\mathrm{LTBI}^{3,4}$.

Contemporary TST and IGRA survey are valuable for epidemiological and clinical assessment as well as in the planning of preventive measure. However, the LTBI prevalence of the general population assessed by the TST and IGRA are not well-known in South Korea, especially in healthy young adults whose age is in twenties. Especially, the prevalence of LTBI has never 
been reported in medical students only in South Korea. Therefore, we investigated the LTBI prevalence among medical students in a medical school in South Korea.

\section{Materials and Methods}

\section{Subjects}

A study to assess the positive rates of the TST and IGRA among medical students was conducted in a medical school, located in Seoul, from March 2010 to March 2012. Students only in second- or third-year class before clerkship course were enrolled. Because this LTBI test was a part of routine health examination in this medical school, written informed consent was waived. A standardized questionnaire included age, gender, past history of any underlying disease including TB, history of contact to patient with TB, and history of Bacillus CalmetteGuérin (BCG) vaccination.

After the completed questionnaire had been obtained, TST and QuantiFERON-TB Gold In-Tube (QFT-GIT; Cellestis Ltd., Carnegie, VIC, Australia) assay were performed at the same time and chest radiograph was taken. This study was approved by the Institutional Review Board of the institution.

\section{Tuberculin skin test}

Each TST was performed by intradermal injection of 2 tuberculin units of purified protein derivative (PPD) RT23 (Statens Serum Institute, Copenhagen, Denmark) into the forearm using the Mantoux technique ${ }^{5}$. Reactions were evaluated 48 to 72 hours after injection, with a positive result defined as an induration of $\geq 10 \mathrm{~mm}$ in the transverse diameter by well-trained personnel.

\section{QuantiFERON-TB Gold In-Tube (QFT-GIT)}

The QFT-GIT assay was performed in 2 stages, according to the manufacturer's instructions. One-milliliter aliquots of blood were drawn directly into three evacuated blood collection tubes, one containing heparin alone (negative control), one containing $\mathrm{T}$ cell mitogen (positive control), and one containing $M$. tuberculosisspecific antigens, including early secreted antigenic tar- get 6 (ESAT-6), culture filtrate protein 10 (CFP-10), and TB7.7 (TB-antigen tube). Following overnight incubation, $200 \mu \mathrm{L}$ of plasma was removed from each well and the concentration of interferon-gamma (IFN- $\gamma$ ) was determined by enzyme-linked immunosorbent assay, with a positive response defined as an antigen-nil IFN- $\gamma$ concentration $\geq 0.35 \mathrm{IU} / \mathrm{mL}^{6}$.

\section{Statistical analysis}

Concordance between results of the TST and QFT-GIT were assessed using kappa $(\kappa)$ coefficients, with $\kappa>0.75$ defined as excellent agreement, $\kappa<0.4$ as poor agreement, and $\kappa$ between 0.4 and 0.75 as fair to good agreement ${ }^{7}$. All statistical analyses were performed using SPSS version 12.0 (SPSS Inc., Chicago, IL, USA).

\section{Results}

\section{Characteristics of subjects}

The study participants consisted of 153 medical students. The mean age of students was $21.9 \pm 0.9$ years, 105 (68.6\%) were male and 132 (86.3\%) had been vaccinated with BCG. One student had a history of pulmonary TB. Four students $(2.6 \%)$ had a history of contact to patient with TB during medical practice while they

Table 1. Clinical characteristics of 153 medical students*

\begin{tabular}{lc}
\hline \multicolumn{1}{c}{ Characteristics } & Medical students ( $n=153)$ \\
\hline Age, yr & $21.9 \pm 0.9$ \\
Gender & \\
$\quad$ Male & $105(68.6)$ \\
Female & $48(31.4)$ \\
Previous TB history & $1(0.6)$ \\
BCG vaccination & \\
Vaccinated & $132(86.3)$ \\
Unvaccinated & $9(5.9)$ \\
Uncertain & $11(7.2)$ \\
Co-morbidity & $1(0.6)$ \\
$\quad$ Glomerulonephritis & \\
\hline
\end{tabular}

*Values are presented as means \pm standard deviations (SD) or number (\%) of subjects.

TB: tuberculosis; BCG: Bacillus Calmette-Guérin. 
Table 2. TST and QFT-GIT results among 153 medical students

\begin{tabular}{lcc}
\hline & \multicolumn{2}{c}{ QFT-GIT } \\
\cline { 2 - 3 } & Positive $(n=8)$ & Negative $(n=145)$ \\
\hline TST & 6 & 17 \\
Positive $(n=23)$ & 2 & 128 \\
Negative $(n=130)$ & & \\
\hline
\end{tabular}

TST: tuberculin skin test; QFT-GIT: QuantiFERON-TB Gold In-Tube.

did preventive measure including wearing $\mathrm{N} 95$ respirator. No abnormal chest radiograph findings were found in all subjects (Table 1).

\section{TST and QFT-GIT results}

Of the 153 subjects, $23(15.0 \%)$ were positive and 130 (85.0\%) were negative on TST. In addition, 8 (5.2\%) were positive and 145 (94.8\%) were negative on QFTGIT. A total of 6 (3.9\%) subjects were positive on both tests (Table 2). The agreement between TST and QFTGIT assay was $\kappa=0.34$. The median value of the QTFGIT of subjects who had positive QTF-GIT responses was $0.97 \mathrm{IU} / \mathrm{mL}$ (range, $0.35 \sim 7.83 \mathrm{IU} / \mathrm{mL}$ ) (Figure 1). Of four students who had a history of contact to patients with TB, only one subject showed positive results of both tests and the other students had negative results of both tests.

We analyzed whether the variables including sex, previous TB history, BCG vaccination and history of contact with TB patients with affect the results of both tests, but no variables were found to influence significantly.

\section{Incidence of tuberculosis during study period}

After a mean follow-up of 17.5 months, none of the medical students have developed active TB.

\section{Discussion}

In present study, LTBI prevalence of medical students in South Korea was investigated using both the TST and

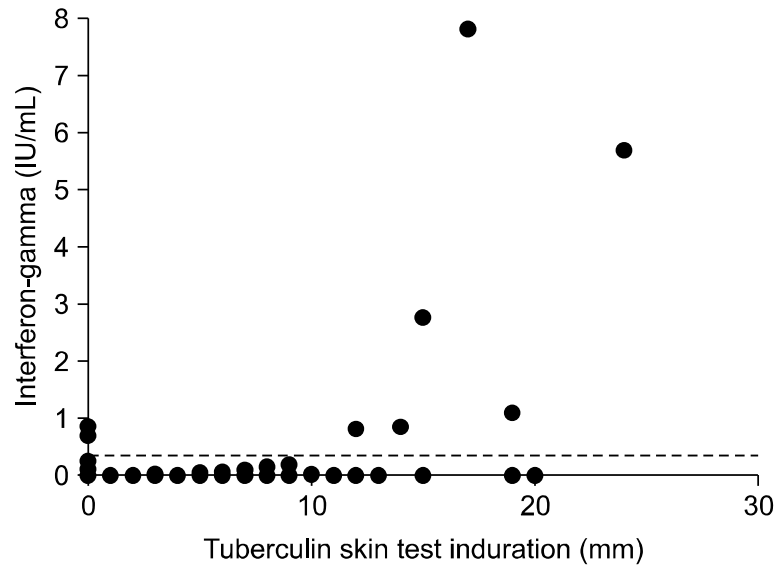

Figure 1. Scatter plot of individual responses of the TST and QFT-GIT in medical students. The dashed line represents the cutoff of $0.35 \mathrm{IU} / \mathrm{mL}$ for interferon-gamma. All points below zero for QTF-GIT results in this figure have been assigned a value of zero. TST: tuberculin skin test; QFT-GIT: QuantiFERON-TB Gold In-Tube.

the QFT-GIT test. We demonstrated that LTBI prevalence was $15.0 \%$ using the TST and $5.2 \%$ using the QFT-GIT among medical students with high rates of BCG vaccination, showing poor agreement between the two tests.

There have been some studies assessing the LTBI prevalence of young general population in South Korea. Kang et al. ${ }^{8}$ investigated the LTBI prevalence in 99 healthy medical students whose median age was 25 years without an identified risk for TB exposure as a control group for their study and reported that 51\% had positive test results for TST and $4 \%$ for QFT-GIT. Similarly, Lee et al. ${ }^{9}$ reported that the TST and QFT-GIT results were positive for $51.5 \%$ and $14.3 \%$, respectively, among 196 newly employed nurses with a mean age of 23.4 years old. Considering the mean ages of participants in these studies, the participants may already be involved in the clerkship course, thus may have many chances to meet the patients with TB. In contrast with these studies, participants in our study did not take clerkship course yet, thus resulting in the low prevalence of LTBI. However, the LTBI prevalence detected by TST seems to be lower in our participants $(15.0 \%)$ than in those of another study in which $28 \%$ individuals had positive TST results among 778 military personnel 
with a mean age 20.0 years who had low risk of TB infection $^{10}$. Socioeconomic factors might have affected on the difference of the LTBI prevalence for each study.

We found that the TST and QFT-GIT showed poor agreement among medical students. The level of agreement between the TST and the QFT-GIT has been reported to generally coincide with the TB prevalence in each country. For example, an excellent agreement between the two tests was observed in Denmark, a low-endemic country, where majority of the people were not BCG vaccinated ${ }^{11}$. In contrast to this, many studies have reported that there was a huge discrepancy between the two tests in South Korea, an intermediated TB-burden country and where BCG vaccination is mandatory $^{8,12,13}$.

It is known that previous BCG vaccination significantly affect the accord of the two tests ${ }^{14,15}$ because the PPD antigen used in the TST leads to false-positive results in individuals vaccinated with BCG. Considering that the finding of one study which revealed BCG vaccination causes positive TST results for 15 years after vaccination $^{16}$ and age of participants in our study, it is reasonable to assume that main reason of poor agreement between the two tests were likely due to the confounding effects of previous BCG vaccination.

The discrepancy we observed could be due to the inaccuracy of the QFT-GIT as well. The ESAT-6, CFP-10, and TB7.7 peptides, which are used in the QFT-GIT assay, do not represent the entire spectrum of antigenicity of $M$. tuberculosis ${ }^{17,18}$. Therefore true LTBI prevalence may be underestimated with IGRA alone ${ }^{19}$. Moreover, short-term within-subject variability has been reported for the IGRA, with variations around the cut-off point for the QFT-GIT $(0.2 \sim 0.7 \mathrm{IU} / \mathrm{mL})$ being a "borderline zone," such that results in this zone should be interpreted with caution ${ }^{20}$. However, only one had the QFT-GIT value of this range $(0.25 \mathrm{IU} / \mathrm{mL})$ among our study population.

Although we investigated LTBI prevalence of distinctive group, the general feature does not seem to be different from other healthy young adults group, because our study population was low-grade medical students who had little chance to contact patients with TB. It would be interesting to investigate LTBI prevalence of the same subject again in several years later after clerkship course.

In conclusion, we found that the prevalence of LTBI among low-grade medical students in a medical college was $15.0 \%$ using the TST and 5.2\% using the QFT-GIT in South Korea. There was poor level of agreement between the two tests.

\section{References}

1. Treatment outcomes of tuberculosis patients through Private-Public Mix collaboration [PPM] in private sector in Korea, 2011 [Internet]. Cheongwon: Korea Centers for Disease Control and Prevention; 2011 [cited 2012 Sep 8]. Available from: http://www.cdc.go.kr/CDC/info/CdcKrInfo0301.jsp?menuIds=HOME001-MNU0001-M NU0036-MNU0037\&q_type $=$ A\&q_value $=2012 \&$ cid $=$ 12798\&pageNum.

2. Kim HJ, Bai GH, Kang MK, Kim SJ, Lee JK, Cho SI, et al. A public-private collaboration model for treatment intervention to improve outcomes in patients with tuberculosis in the private sector. Tuberc Respir Dis 2009; 66:349-57.

3. Horsburgh CR Jr, Rubin EJ. Clinical practice: latent tuberculosis infection in the United States. N Engl J Med 2011;364:1441-8.

4. Schablon A, Diel R, Diner G, Anske U, Pankow W, Ringshausen FC, et al. Specificity of a whole blood IGRA in German nursing students. BMC Infect Dis 2011;11:245.

5. Sokal JE. Editorial: measurement of delayed skin-test responses. N Engl J Med 1975;293:501-2.

6. Mori T, Sakatani M, Yamagishi F, Takashima T, Kawabe Y, Nagao K, et al. Specific detection of tuberculosis infection: an interferon-gamma-based assay using new antigens. Am J Respir Crit Care Med 2004;170: 59-64.

7. Landis JR, Koch GG. The measurement of observer agreement for categorical data. Biometrics 1977;33:15974 .

8. Kang YA, Lee HW, Yoon HI, Cho B, Han SK, Shim YS, et al. Discrepancy between the tuberculin skin test and the whole-blood interferon gamma assay for the diagnosis of latent tuberculosis infection in an intermediate tuberculosis-burden country. JAMA 2005;293: 2756-61. 
9. Lee K, Han MK, Choi HR, Choi CM, Oh YM, Lee SD, et al. Annual incidence of latent tuberculosis infection among newly employed nurses at a tertiary care university hospital. Infect Control Hosp Epidemiol 2009; 30:1218-22.

10. Choi CM, Kang CI, Kim DH, Kim CH, Kim HJ, Lee CH, et al. The role of TST in the diagnosis of latent tuberculosis infection among military personnel in South Korea. Int J Tuberc Lung Dis 2006;10:1342-6.

11. Brock I, Weldingh $\mathrm{K}$, Lillebaek $\mathrm{T}$, Follmann $\mathrm{F}$, Andersen P. Comparison of tuberculin skin test and new specific blood test in tuberculosis contacts. Am J Respir Crit Care Med 2004;170:65-9.

12. Choi JC, Shin JW, Kim JY, Park IW, Choi BW, Lee MK, The effect of previous tuberculin skin test on the follow-up examination of whole-blood interferon-gamma assay in the screening for latent tuberculosis infection. Chest 2008;133:1415-20.

13. Lee KJ, Kang YA, Kim YM, Cho SN, Moon JW, Park MS, et al. Screening for latent tuberculosis infection in South Korean healthcare workers using a tuberculin skin test and whole blood interferon-gamma assay. Scand J Infect Dis 2010;42:672-8.

14. Huang YW, Shen GH, Lee JJ, Yang WT. Latent tuberculosis infection among close contacts of multidrug-resistant tuberculosis patients in central Taiwan. Int J Tuberc Lung Dis 2010;14:1430-5.
15. Shalabi NM, Houssen ME. Discrepancy between the tuberculin skin test and the levels of serum interferon-gamma in the diagnosis of tubercular infection in contacts. Clin Biochem 2009; 42:1596-601.

16. Wang L, Turner MO, Elwood RK, Schulzer M, FitzGerald JM. A meta-analysis of the effect of Bacille Calmette Guérin vaccination on tuberculin skin test measurements. Thorax 2002;57:804-9.

17. Demangel C, Brodin P, Cockle PJ, Brosch R, Majlessi L, Leclerc C, et al. Cell envelope protein PPE68 contributes to Mycobacterium tuberculosis RD1 immunogenicity independently of a 10-kilodalton culture filtrate protein and ESAT-6. Infect Immun 2004;72: 2170-6.

18. Liu XQ, Dosanjh D, Varia H, Ewer K, Cockle P, Pasvol $\mathrm{G}$, et al. Evaluation of T-cell responses to novel RD1and RD2-encoded Mycobacterium tuberculosis gene products for specific detection of human tuberculosis infection. Infect Immun 2004;72:2574-81.

19. Lew WJ, Jung YJ, Song JW, Jang YM, Kim HJ, Oh YM, et al. Combined use of QuantiFERON-TB Gold assay and chest computed tomography in a tuberculosis outbreak. Int J Tuberc Lung Dis 2009;13:633-9.

20. van Zyl-Smit RN, Pai M, Peprah K, Meldau R, Kieck J, Juritz J, et al. Within-subject variability and boosting of T-cell interferon-gamma responses after tuberculin skin testing. Am J Respir Crit Care Med 2009;180:49-58. 